\title{
LAS CONSECUENCIAS ELECTORALES DE LA MOVILIZACIÓN TERRITORIAL Las estrategias del Partido Popular en la campaña de 1996
}

\author{
HENAR CRIADO OLMOS* \\ IESA-CSIC. Córdoba
}

\begin{tabular}{ll}
\hline PALABRAS CLAVE ADICIONALES & ADDITIONAL KEYWORDS \\
Circunscripción, Campaña electoral, Partido & District, Electoral Campaign, Political Party, \\
político, Mítines, Gasto descentralizado. & Rallies, Decentralized Expenditure
\end{tabular}

político, Mítines, Gasto descentralizado.

Rallies, Decentralized Expenditure

\begin{abstract}
RESUMEN. En este artículo se analiza el impacto de las estrategias de movilización territorial en el voto de los electores. En concreto, se estudian los efectos de las visitas del candidato del partido, el gasto descentralizado y la inclusión de figuras conocidas en las listas provinciales, en el voto de los electores que residen en estas circunscripciones. Frente a los trabajos que analizan el impacto de las características individuales de los electores, se ha estimado un modelo multinivel que permite comprobar el efecto de las variables de movilización de los partidos en las circunscripciones.
\end{abstract}

SUMMARY. In this paper I analyze the effects of the territorial mobilization strategies on the vote. More concretely, I have analyzed the effects on the vote in each district of the candidates' rallies, the party's decentralized expenditure and the inclusion of known candidates in the party's district list. In order to do this, I have estimated a multilevel model to test the impact of aggregate mobilization variables at the district level controlling by the voters' individual characteristics.

E-mail: hcriado@iesaa.csic.es

*Becaria del IESA-CSIC (Córdoba) y estudiante de doctorado de la Fundación Juan March.

Revista Internacional de Sociología (RIS)

Tercera Época, $\mathrm{n}^{\circ} 32$, Mayo-Agosto, 2002, pp. 103-124. 


\section{INTRODUCCIÓN}

Durante las campañas electorales, los partidos no distribuyen sus recursos de manera homogénea entre las diferentes circunscripciones electorales. No en todas las circunscripciones se invierte el mismo dinero y no todas las provincias reciben la visita del candidato del partido. Es razonable pensar que los partidos distribuyen sus esfuerzos electorales entre las circunscripciones para maximizar su probabilidad de ganar. La cuestión es si consiguen alcanzar su objetivo. ¿Qué consecuencias tienen los esfuerzos realizados por los partidos en las circunscripciones sobre el voto de los ciudadanos?

En este trabajo responderé a esa cuestión, analizando las estrategias de movilización territorial del PP en las elecciones generales de 1996. El artículo se estructurará de la siguiente manera. En el apartado siguiente discuto cómo la literatura de la movilización electoral ha respondido a esta cuestión, y cuáles son las limitaciones de este enfoque. A continuación, en el apartado tercero presento un modelo logit multinivel y los datos que empleo para analizar el impacto de las estrategias agregadas de la movilización sobre el comportamiento individual de los electores. En el apartado cuarto muestro los resultados empíricos del modelo.

\section{El impacto de la movilización territorial en el voto}

Muchos de los estudios sobre comportamiento electoral analizan el impacto de las características individuales en el voto. La mayoría de estos estudios pueden agruparse en torno a dos tradiciones. Por un lado, una tradición más europea que analiza [el impacto] de las características sociodemográficas, especialmente la clase social, en el voto (Lipset y Rokkan, 1967; González, 1996; Torcal y Chhibber, 1997; Caínzos, 2002), y por el otro una tradición más norteamericana que analiza los efectos de las características psicosociales (actitudes) en el voto (Campbell, Converse, Miller y Stokes, 1960; Gunther y Montero, 1994)'. Frente a este tipo de análisis, la escuela de la movilización destaca la influencia de las estrategias desarrolladas por los partidos en el comportamiento electoral de los ciudadanos. Estos trabajos pueden dividirse a su vez en dos grupos. En el primer grupo estarían los trabajos que analizan cómo el hecho de que un partido contacte directamente con sus votantes aumenta la probabilidad del voto a este

\footnotetext{
'Estos son sólo algunos de los trabajos que analizan el impacto de diversas características individuales de los ciudadanos tanto en su probabilidad de votar como en la dirección de su voto. No se trata, como es obvio, de una lista exhaustiva. Existen muchos más trabajos que comparten esta perspectiva.
} 
partido (Huckfeldt y Sprague,1992; Rosenstone y Hansen, 1993; Wielhouver y Lockerbie, 1994). El segundo grupo lo integran aquellos trabajos que estudian cómo la distribución territorial de recursos y esfuerzos movilizadores de los partidos influye en el voto (Cox y Munger, 1989; Cox, Rosenbluth y Thies 1998; Goldstein, 1999). El análisis que llevaré a cabo en este artículo se adscribe más bien a este segundo grupo de trabajos acerca de la movilización electoral a nivel territorial. Analizo cómo tres estrategias diferentes de movilización del PP afectan al voto durante la campaña de 1996. Estas estrategias son: el dinero que se destina descentralizadamente a las circunscripciones, los mítines realizados por Aznar, y la inclusión de figuras muy relevantes del partido encabezando las listas provinciales. Como puede observarse, se trata de estrategias de movilización territorial. Quedan excluidas del análisis las estrategias de movilización individual.

En general, los trabajos que se encuadran dentro de la escuela de la movilización se han ocupado más de explicar cuáles son los criterios que los partidos utilizan para esforzarse más en unas circunscripciones que en otras, o en unas elecciones que en otras, que en el análisis del impacto de estas estrategias en el voto. Este tipo de estudios tienen, al menos, dos problemas: no distinguen entre perfiles de votantes, algo que parece relevante para evaluar el impacto de las estrategias electorales y no establecen los mecanismos que conectan la movilización de los partidos y el voto. Además de estos dos problemas, se podría achacar igualmente a estos estudios la ausencia de un diseño metodológico adecuado para medir el impacto de las variables agregadas de movilización en el comportamiento individual de los electores. En un apartado posterior me referiré con mayor detenimiento a este último problema. A continuación argumentaré que los dos primeros problemas pueden ser al menos paliados combinando esta literatura con los análisis referidos a las campañas electorales.

Normalmente, se ha considerado que la escuela de la movilización era la única que resaltaba la importancia de la oferta partidista en el estudio del voto. Pero la idea de que las estrategias de movilización electoral desarrolladas por los partidos incentivan el voto de los ciudadanos está implícita también en la literatura sobre campañas electorales. Los trabajos que pertenecen a este enfoque analizan el impacto que las campañas tienen sobre el voto de los ciudadanos (Lazarsfeld, Berelson y Gaudet, 1948; Holbrook, 1996). Obviamente, si las campañas electorales influyen en el voto es porque las estrategias electorales desarrolladas por los partidos en la campaña condicionan el comportamiento de los electores. Desgraciadamente, ambos programas de investigación -el de los efectos de las campañas y el de la movilización electoral-, a pesar de compartir una perspectiva muy similar, han llevado líneas de investigación diferentes.

Como ya he dicho, en los estudios de movilización no se distingue entre perfiles de votantes para analizar el impacto de las estrategias de los partidos (Cox y 


\section{RIS}

Munger, 1989; Wielhouber y Lockerbie, 1994; Goldstein, 1999)2. Simplemente, analizan el impacto de la movilización en el voto de los electores sin distinguir, por ejemplo, su predisposición inicial. Sin embargo, es razonable pensar que dado que la movilización es la referida a la campaña electoral, su efecto sobre el voto será mayor en los votantes indecisos, aquellos que han decidido su voto durante la campaña. Por el contrario, las estrategias de movilización apenas influirán en votantes muy identificados con sus partidos, aquellos que tenían decidido su voto con anterioridad a la campaña electoral.

El segundo problema de la literatura de movilización es que no especifica claramente los mecanismos que explican por qué la movilización electoral incentiva el voto de los electores. En general, se limita simplemente a demostrar la correlación entre ambas variables. Y ello sin analizar en profundidad los mecanismos que conectan las estrategias de los partidos y el voto de los ciudadanos.

A diferencia de la escuela de la movilización, los trabajos que analizan el efecto de las campañas electorales no se ven afectados por estos dos problemas. En primer lugar, los trabajos sobre campañas electorales sí distinguen entre perfiles de electores. De hecho, una de las primeras aportaciones concluyentes de los estudios de campañas al análisis del comportamiento electoral fue que las campañas electorales influían sólo en aquellos electores sin identidades partidistas e ideológicas estables (Lazarsfeld, Berelson y Gaudet, 1948). En segundo lugar, los estudios sobre campañas sí han analizado los mecanismos que conectan la movilización electoral y el voto. En los trabajos sobre campañas se distinguen tres efectos de las estrategias de los partidos durante la campaña electoral: el efecto activación, el efecto conversión y el efecto de refuerzo (Lazar-

\footnotetext{
${ }^{2}$ Otra razón por la que es importante seleccionar a los votantes indecisos, aquellos que decidieron su voto durante la campaña es de carácter metodológico. Uno de los estudios que analizan el impacto de la movilización en la participación es la dirección de la causalidad: ¿tienen los ciudadanos movilizados mayor probabilidad de votar por un determinado partido o, por el contrario, es que los partidos movilizan a aquellos cuya probabilidad de votar por ellos era inicialmente mayor? Si se da este segundo caso, al analizar el impacto de la movilización se produciría un sesgo de selección. Esto es, estamos asumiendo que los casos de la variable de movilización son seleccionados aleatoriamente, cuando en realidad no es así. El modelo, entonces, asumiría que la esperanza del error estocástico es 0, cuando en realidad no lo es (Goldstein, 1999: 112; Gujarati 1997: 311-2; Peña, 2001: 551-560). Una manera de salvar este problema metodológico es seleccionar solamente aquellos votantes que dicen haber decidido su voto durante la campaña. Es plausible que esta submuestra de electores reúna a votantes que inicialmente no tenían una alta probabilidad de votar a un determinado partido, ya que ellos mismos declaran haber decidido su voto durante la campaña. Además, es razonable pensar que los partidos carecen de la información suficiente para seleccionar a sus votantes indecisos dentro de cada provincia. Por ello, el riesgo de que con esta submuestra continúe existiendo un sesgo de selección disminuye.
} 
sfeld, Berelson y Gaudet, 1944; Finkel, 1993; Mellizo-Soto 2000: 2-5) ${ }^{3}$. De estos tres, la mayoría de los autores coincide en destacar el efecto activador de la movilización. De hecho, se considera que la campaña tiene fundamentalmente un efecto activador del voto. Esto es, las estrategias de los partidos servirían para activar las predisposiciones políticas previas o latentes de los electores. El efecto conversión de la movilización es, sin embargo, mucho más discutido. En general, se supone que las actividades de los partidos durante la campaña no tienen un gran impacto en el cambio de voto. El efecto refuerzo, por su parte, es considerablemente más trivial que el activador, ya que consiste simplemente en evitar que los electores favorables hacia un partido opten por el partido rival.

De acuerdo con estos argumentos, se puede concluir que los trabajos sobre campañas electorales analizan más en profundidad que los artículos sobre movilización electoral el perfil de votantes a los que influye la campaña ỳ los mecanismos que explican las razones de esta influencia. Sin embargo, esto no implica que los estudios sobre campañas electorales no tengan limitaciones. Una de estas limitaciones es que no definen claramente la conexión entre las acciones de los partidos durante la campaña y el voto de los ciudadanos. Se limitan a analizar el efecto de las acciones de los partidos de manera indirecta a través del interés y el seguimiento por los votantes de la campaña. De hecho, es muy frecuente que se estudie el impacto de las campañas midiendo la probabilidad de votar a un determinado partido antes y después de la campaña, analizando si el seguimiento de la misma a través de los medios de comunicación explica los cambios entre un momento y otro. El problema de este tipo de análisis es que no estudian en profundidad la conexión entre las estrategias de campaña de los partidos y el voto de los electores. No sabemos si la probabilidad de votar al partido cambia por la imagen de confianza que el líder ha sabido transmitir, o por haber transmitido una imagen de eficacia, o bien, por ejemplo, por los temas en los que el partido ha basado su discurso, $o$, porque el candidato que se presenta por su provincia le parece una persona muy implicada en los problemas de su región o, finalmente, por la gran difusión de que han disfrutado todas estas cuestiones en las radios, la prensa o la televisión nacionales y locales.

Para superar las limitaciones de ambas tradiciones, en este análisis combino las aportaciones de la escuela de la movilización con la de los efectos de las campañas. Para ello, en primer lugar, analizo si la movilización del PP durante la campaña de 1996 tuvo algún efecto sobre dos tipos de votantes indecisos: aquellos que declaran haber decidido votar durante la campaña y aquellos que

\footnotetext{
${ }^{3}$ Para un análisis sobre el impacto de la campaña de 1993 en el voto de los electores indecisos ver Barreiro y Sánchez-Cuenca (1998).
} 
declaran haber decidido a qué partido votar durante la misma. Al distinguir entre perfiles de indecisos profundizo no sólo en los diferentes efectos de la movilización entre perfiles de votantes sino también en los mecanismos - activación o conversión - que explican el por qué de estos efectos. Además, intento establecer directamente la conexión entre las estrategias de movilización de las elites y el voto de los electores.

\section{EL MODELO}

Como hemos visto, los trabajos acerca de la movilización se centran más en explicar los criterios por los cuales los partidos asignan sus recursos que los efectos de estas asignaciones de recursos sobre el voto. De hecho, muchos de ellos se limitan a correlacionar de manera agregada los recursos asignados a cada circunscripción con el voto al partido en esa circunscripción (Cox y Munger, 1989; Cox, Rosenbluth y Thies, 1994). Sin embargo, para responder a esta pregunta con rigor es necesario añadir variables contextuales de movilización agregada al nivel de distrito a encuestas electorales individuales. De esta forma se comprueba cómo la movilización electoral en las circunscripciones influye en la participación de los electores controlando por sus características individuales (Jones y Bullen, 1994; Goldstein, 1995; Goldstein, 1999).

Normalmente, los estudios de comportamiento electoral analizan el efecto de las características individuales de los ciudadanos en su probabilidad de votar, o de votar por un determinado partido (Lipset y Rokkan, 1967; Campbell, Converse, Miller y Stokes, 1960; Verba, Nie y Kim, 1978; Wolfinger y Rosenstone, 1980). Los trabajos que analizan la influencia del contexto, por su parte, suelen concentrarse en el estudio de variables de carácter institucional, como el sistema electoral (Jackman, 1987; Anduiza, 1999). No obstante, recientemente numerosos

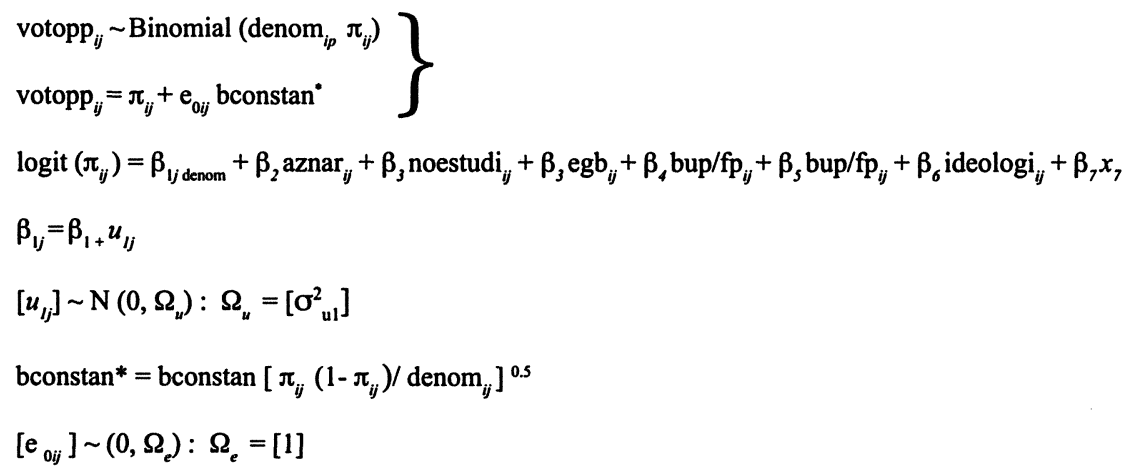


estudios han incluido dentro de las variables contextuales variables referidas a la movilización electoral (Stoker y Bowers, 2002; Marsh, 2002; Johnson, Shively y Stein, 2002). En este modelo, las variables contextuales son variables que miden el impacto de la movilización agregada en las circunscripciones electorales.

El modelo general que he estimado es el siguiente. La variable dependiente es el voto al PP de los electores indecisos. Se ha introducido en los modelos un conjunto de variables independientes referidas a características individuales de los votantes; en concreto, autoubicación ideológica (ideologi), valoración del líder del partido (candidat) y una variable referida al sentimiento de representación de los intereses del elector por el partido (represe). Como variable sociodemográfica he incluido educación. Se trata de una variable categórica. La categoría de referencia en todos los modelos son aquellos que han cursado algún tipo de estudio universitario. El resto de categorías son: sin estudios (noestudi), estudios primarios (egb), bachillerato o formación profesional (bupofp).

Las variables agregadas de movilización, por su parte, son: gasto, personas conocidas incluidas en las listas provinciales, mítines del candidato, y un indicador agregado de todas estas actividades. En cada modelo se incluye una de estas actividades movilizadoras. En el modelo general, la variable de movilización corresponde a la variable $X_{7}$. De manera muy resumida puede decirse que los criterios con los que el PP distribuyó sus esfuerzos movilizadores entre las circunscripciones varían dependiendo de la actividad movilizadora de que se trate. El PP concentró sus mítines en las provincias con mayor número de escaños y donde la competitividad esperada era mayor, gastó más en provincias con menor número de escaños - provincias que son, por otra parte, sus "graneros" electorales tradicionales- y situó a sus candidatos más importantes siguiendo no criterios estratégicos, sino más bien criterios referidos a las tradiciones organizativas del partido. En resumen, de las tres actividades movilizadoras incluidas en el modelo es el diseño de los mítines de José María Aznar la que se realizó siguiendo criterios de carácter más estratégico. En las otras dos primaron otras cuestiones de carácter más organizativo (Criado, 2002).

Para estimar un modelo que incluye variables contextuales e individuales utilizaré un modelo multinivel (multilevel) (Goldstein, 1995: 5-36; Jones y Bullen, 1994: 252-255). Los modelos multinivel permiten estimar simultáneamente varios niveles en los datos, lo cual mejora la estimación tanto a nivel individual como a nivel contextual. En este caso los dos niveles de análisis son las circunscripciones y los individuos.

Normalmente, los modelos de un solo nivel analizan bien la variación entre circunscripciones a nivel agregado. Otro tipo de estudios de un solo nivel analiza la variación entre individuos, destacando la importancia de las características individuales en su probabilidad de votar. A los modelos que estudian la variación a nivel agregado se les suele criticar por los riesgos de falacias ecológicas. Por su parte, los modelos que analizan las diferencias entre individuos no adolecen de 
RIS

este problema, pero a menudo no pueden contrastar, excepto de manera indirecta, los efectos sobre el comportamiento individual de variables institucionales. Por el contrario, un modelo multinivel me permite analizar no sólo ambos tipos de variación -entre circuncripciones y entre individuos-, sino, lo que es más importante, la interacción de las variables agregadas con las características individuales de la población (Jones y Bullen, 1994: 256-59; Goldstein, 1995:23-25).

Además de estas razones generales hay un problema específico de micro numerosidad de la muestra en los datos que aconseja la utilización de este modelo. En algunas de las provincias de la encuesta que he utilizado, el número de votantes indecisos es muy pequeño. En los modelos tradicionales la movilización a nivel de circunscripción es considerada como un atributo de cada individuo. Las circunscripciones y los individuos son tratados como observaciones equivalentes. Cuando el número de observaciones en cada circunscripción es muy pequeño, como sucede en este caso, la variación dentro de cada circunscripción se confunde con la variación entre circunscripciones, por lo que los resultados pueden estar sesgados. A continuación desarrollo más formalmente por qué un modelo multinivel produce mejores resultados para muestra pequeñas en uno de los niveles de análisis (Jones y Bullen, 1994: 260-66).

La especificación de un modelo multinivel, siguiendo la notación de Jones y Bullen (1994), es la siguiente:

$$
Y_{i j}=\beta_{0 j} X_{0 i j}+\beta_{1} X_{1 i j}+\epsilon_{i j} X_{0 i j}
$$

En este modelo, el subíndice i se refiere al nivel 1 (en nuestro caso, individuos), $y$ el subíndice $\mathrm{j}$, al nivel 2 (en nuestro análisis, circunscripciones). Es importante destacar que al intercepto se le deja variar al nivel 2, esto es, entre circunscripciones. De manera que:

$$
\beta_{0 j}=\beta_{0}+\mu_{0 j}
$$

El modelo multinivel completo es, por lo tanto:

$$
Y_{i j}=\beta_{0} X_{0 i j}+\beta_{1} X_{1 i j}+\left(\mu_{0 j} X_{0 i j}+\epsilon_{i j} X_{0 i j}\right)
$$

La mayor utilidad para el análisis derivada de la utilización de un modelo multinivel es que el intercepto de una determinada circunscripción es una combinación ponderada del intercepto de la circunscripción y el intercepto general.

Es decir:

$$
\beta_{0 j}=p_{j} \beta_{0 j}^{*}+\left(1-p_{j}\right) \beta_{0}
$$


Nótese que el intercepto de cada circunscripción se pondera con el intercepto general (Jones y Bullen, 1994: 260). Esta especificación me proporciona un estimador más robusto para las circunscripciones donde el número de individuos es pequeño. La media de las observaciones dentro de cada circunscripción se pondera con la media de todas las observaciones. De este modo se contrarresta que las observaciones dentro de una circunscripción sean pocas y heterogéneas evitando que la variación dentro de cada circunscripción se confunda con la variación entre circunscripciones.

En cuanto a los datos utilizados, pertenecen a la encuesta post-electoral del CIS de marzo de 1996, estudio número 2.210. Se trata de una encuesta sobre comportamiento electoral a nivel nacional. El tamaño de la muestra es de 5.300 individuos. Incluye variables referidas al comportamiento electoral de los votantes, y variables acerca de las características sociodemográficas y actitudes políticas de los individuos. Las variables agregadas referidas a las provincias movilizadas se han construido a partir de la información de prensa y de gerencia del PP.

\section{ELEFECTO DE LAMOVILIZACIÓN SOBRE LOS VOTANTES INDECISOS. LA VARIABLE DEPENDIENTE}

Como ya he mencionado en varios epígrafes del artículo, el objetivo fundamental de este trabajo es analizar el efecto en los votantes indecisos de la movilización territorial del PP durante la campaña electoral. Esto es, en aquellos que declaran haber decidido su voto durante este período. He creado tres perfiles diferentes de votantes indecisos. En primer lugar, he seleccionado de entre todos los votantes aquellos que declaran haber decidido votar o a qué partido votar durante la campaña. Es decir, todos los votantes indecisos. La codificación de las variable es la siguiente. El valor 1 corresponde a todos aquellos que decidieron o bien votar o bien la dirección de su voto durante la campaña y votaron al PP. El valor 0 lo forman aquellos que durante la campaña deciden o bien no votar o votar por un partido distinto al PP.

En el segundo perfil de votantes he seleccionado de entre todos los indecisos aquellos que dudaban entre votar y abstenerse, y finalmente decidieron votar por el PP. Con este perfil de votantes compruebo si la movilización tuvo un efecto activador en este subgrupo. La codificación de la variable es la siguiente. El valor 1 corresponde a aquellos que deciden votar durante la campaña y lo hacen por el $\mathrm{PP}$. El valor 0 corresponde a todos aquellos que decidieron durante la campaña no votar o votar por un partido distinto al PP.

Por último, he seleccionado de todos los indecisos a los que no sabían por qué partido votar y finalmente votaron por el PP. Con este perfil de votantes pretendo analizar el efecto "conversor" de la campaña electoral. El valor 1 corresponde a aquellos que dudaban a qué partido votar, y finalmente se deciden por el PP. El 
valor 0 reúne a aquellos que durante la campaña decidieron votar a un partido distinto al PP, o no votar.

En resumen, hay tres indicadores diferentes de voto al PP. En el primero se incluye a todos los indecisos que decidieron durante la campaña votar por el PP. En el segundo indicador se incluyen, de todos los indecisos, sólo a aquellos que dudaban entre abstenerse o no y finalmente votaron al PP. El último indicador lo forman todos los indecisos que sí pensaban votar pero no sabían a qué partido. La desagregación de estos tres diferentes perfiles de votantes indecisos me permite comprobar si la movilización tuvo un efecto activador del voto o formador de preferencias. A continuación describiré brevemente la distribución de frecuencias de estos indicadores.

De acuerdo con el estudio CIS 2.210, del $87 \%$ de los electores que declaran haber votado en las elecciones de $1996^{4}$ el porcentaje total de indecisos (votantes que declaran haber decidido votar y/o la dirección de su voto en el transcurso de la campaña electoral) es del $14 \%$. De estos indecisos, un $8 \%$ decidió durante la campaña ir a votar, y un $11 \%$ decidió en ese mismo período a qué partido votars. En cuanto a las diferencias entre partidos, del total de indecisos, un $30 \%$ votó al PSOE y un $20 \%$, al PP. Por su parte, de entre los que declaran haber decidido durante la campaña ir a votar -indecisos activados- el 30\% votó al PSOE, mientras que el $19 \%$ lo hizo por el PP. Finalmente, de entre aquellos que declararon haber decidido durante la campaña a qué partido votar, el $31 \%$ se inclinó finalmente por el PSOE, y el 20\%, por el PP. Parece claro que dentro de los votantes del PP el porcentaje de indecisos fue menor que en los votantes del PSOE ${ }^{6}$. O dicho de otro modo, de todos los votantes indecisos un porcentaje mayor terminó optando por el PSOE en lugar del PP.

\section{RESULTADOS}

Para analizar cuál fue la incidencia de la movilización electoral sobre los electores que decidieron su voto en el transcurso de la campaña, comenzaré presentando los resultados de un análisis bivariado entre los diferentes indicadores de movilización y las variables de participación electoral.

\footnotetext{
${ }^{4}$ El porcentaje real de voto en estas elecciones fue de $79 \%$. Normalmente, hay una cierta tendencia de los encuestados a ocultar que se abstuvieron. Esto produce un sesgo en la encuesta en la estimación del voto.

${ }^{5} \mathrm{El}$ porcentaje total de indecisos es un $14 \%$ y no un $19 \%$ porque en algunos casos aquellos que decidieron durante la campaña ir a votar decidieron al mismo tiempo a qué partido votar.

${ }^{6}$ De todos los que declaran haber votado al PSOE un $14 \%$ lo decidieron durante la campaña mientras que de los votantes del PP solo un $10 \%$.
} 
Como puede observarse en la tabla 1 , el porcentaje de indecisos que declaran haber votado al PP es 5 puntos mayor en las provincias que contaron con la presencia de Aznar en sus mítines que en el resto de provincias (de un $23 \%$ a un $18 \%$ ). Si seleccionamos dentro de todos los indecisos aquellos que decidieron si votar durante la campaña y finalmente votaron al PP, observamos que el impacto de los mítines del candidato es todavía mayor. El porcentaje que finalmente votó por el PP es del $25 \%$ en las visitadas por Aznar y en las no visitadas, de un $16 \%$. Sin embargo, el impacto de la visita de Aznar es bastante menor para aquellos que decidieron durante la campaña a qué partido votar y votaron finalmente al PP. El porcentaje de indecisos que dudaban a qué partido votar y finalmente votaron al PP en las provincias visitadas por Aznar es $21 \%$ y en las que no, del $18 \%$.

A la luz de estos resultados, parece evidente que el efecto de la movilización estratégica, en concreto de los mítines, es mayor entre aquellos que decidieron durante la campaña votar, y finalmente votaron al PP. Es decir, la movilización electoral, como ha sido argumentado sistemáticamente por la literatura, tiene sobre todo un efecto de activación del voto.

Otra cuestión relevante de los resultados que aparecen en la tabla 1 son las diferencias, no ya entre perfiles de indecisos, sino entre distintas formas de movilización electoral. Los mítines del candidato son los que tienen una influencia mayor en el porcentaje de voto. Resulta muy interesante que los mítines sean la actividad movilizadora con mayor impacto en el voto. Como ya mencioné en el epígrafe anterior, se trata de la forma de movilización que se diseñó más estratégicamente desde el PP. De hecho José María Aznar visitó las provincias donde la elección era más competida (Criado, 2002). El resto de las actividades movilizadoras del PP tuvieron un impacto menor en el voto, como es el caso de la inclusión de figuras conocidas del partido en las listas provinciales. La diferencia en porcentaje de voto al PP entre las provincias que contaron con figuras conocidas y las que no es apenas de uno o dos puntos a favor de las primeras para todos los perfiles de indecisos. Las diferencias son algo mayores en el caso del gasto descentralizado.

Además de analizar el efecto de cada una de estas tres actividades movilizadoras por separado, he empleado un indicador global de movilización. Este indicador se construye mediante la suma de las tres actividades de movilización incluidas en el modelo, de manera que el valor 0 corresponde a las circunscripciones donde no se ha realizado ningún tipo de actividad movilizadora, y los valores $1,2, \mathrm{y}$ 3 , corresponden a las circunscripciones donde se han llevado a cabo 1, 2, ó 3 actividades movilizadoras, respectivamente. En la tabla 2 aparecen los resultados de un análisis bivariado de este indicador con el porcentaje de votos al PP por perfiles de indecisos. Como puede observarse, los resultados de la tabla 2. son coherentes con los observados en la tabla anterior. El porcentaje de indecisos que finalmente votaron por el PP es mayor a medida que aumenta la intensidad de la movilización. Además, el impacto de la movilización es mayor para aquellos que 


\section{RIS}

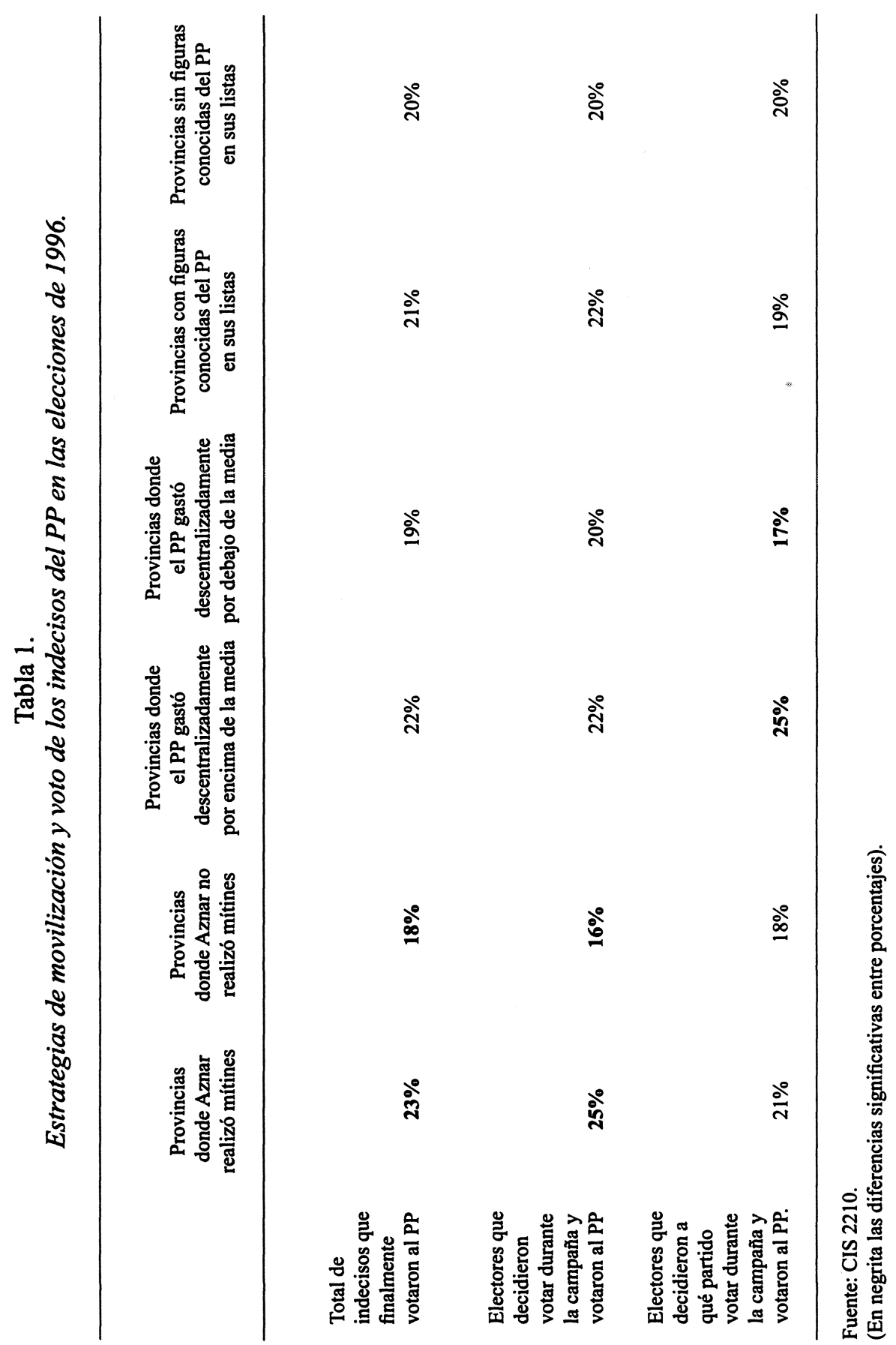


Tabla 2.

Indicador agregado de movilización y voto de los indecisos al PP en las elecciones de 1996.

\begin{tabular}{|c|c|c|c|c|}
\hline & $\begin{array}{c}\text { Provincias } \\
\text { donde no se } \\
\text { movilizó }\end{array}$ & $\begin{array}{c}\text { Provincias } \\
\text { donde se realizó } \\
\text { una actividad } \\
\text { movilizadora }\end{array}$ & $\begin{array}{l}\text { Provincias donde } \\
\text { se realizaron dos } \\
\text { actividades } \\
\text { movilizadoras }\end{array}$ & $\begin{array}{l}\text { Provincias donde } \\
\text { se realizaron tres } \\
\text { actividades } \\
\text { movilizadoras }\end{array}$ \\
\hline $\begin{array}{l}\text { Total de } \\
\text { indecisos que } \\
\text { finalmente } \\
\text { votaron al PP }\end{array}$ & $15 \%$ & $21 \%$ & $22 \%$ & $23 \%$ \\
\hline $\begin{array}{l}\text { Electores que } \\
\text { decidieron } \\
\text { votar durante } \\
\text { la campaña y } \\
\text { votaron al PP }\end{array}$ & $15 \%$ & $21 \%$ & $23 \%$ & $33 \%$ \\
\hline $\begin{array}{l}\text { Electores que } \\
\text { decidieron a } \\
\text { qué partido } \\
\text { votar durante } \\
\text { la campaña y } \\
\text { votaron al PP }\end{array}$ & $17 \%$ & $20 \%$ & $20 \%$ & $23 \%$ \\
\hline
\end{tabular}

Fuente: CIS 2.210.

decidieron durante la campaña si ir a votar. Esto es, la movilización tiene sobre todo un efecto activador del voto.

En estos resultados del análisis bivariado se aprecian ciertos patrones en la influencia de las actividades movilizadoras en el voto al PP. No obstante, estos resultados no son en modo alguno concluyentes. Es necesario realizar un análisis estadístico más complejo que introduzca diferentes niveles de variación en los datos. A continuación se muestran los resultados obtenidos del análisis multinivel multivariable.

El impacto de la movilización territorial en la participación electoral. Un análisis multinivel

Como comentaba en el apartado metodológico, un modelo multinivel es el más adecuado para medir el efecto de las variables agregadas de movilización en el comportamiento de los electores controlando por sus características individuales. La utilización de un análisis multinivel es necesaria, también, debido a que 
el tamaño de la muestra utilizada es bastante pequeño. A continuación presento los resultados de los modelos multinivel. No he incluido en el análisis uno de los perfiles de indecisos que sí aparecía en el análisis bivariado. Se trata de los electores que deciden a qué partido votar durante la campaña y votaron por el PP. La razón de la exclusión de este perfil de indecisos son los resultados obtenidos en el análisis bivariado, o mejor dicho la ausencia de resultados de este análisis. En los porcentajes bivariados se observaba que la movilización no tenía apenas efectos en los indecisos que deciden a qué partido votar durante la campaña. Para hacer la exposición de los efectos de la movilización de manera más estilizada los he excluido del análisis multinivel. En las tablas 3 y 4 pueden observarse los resultados de los modelos multinivel.

En la tabla 3 aparecen los resultados de los modelos multinivel para todos los electores indecisos que, finalmente, votaron por el PP. Se han estimado cuatro modelos diferentes. En cada modelo se ha introducido una de las variables de movilización. No se han incluido las cuatro variables agregadas de movilización en un solo modelo para evitar problemas de colinealidad. Las variables individuales son las mismas para todos los modelos: autoubicación ideológica, valoración de Aznar, representación de los intereses del elector por el partido y educación.

Como era de esperar, los electores indecisos de ideología de derechas, que valoraban positivamente a Aznar y que sentían representados sus intereses por este partido tuvieron una mayor probabilidad de votarle. También le votaron más los indecisos con mayor nivel educativo. Nótese que el tamaño y el signo de los coeficientes de las variables individuales es muy parecido en los cuatro modelos. No obstante, las variables individuales explican sólo una parte del voto de los indecisos por el PP. La variación del voto al PP se da no sólo entre individuos sino también entre provincias. Esto es, los individuos que viven en determinadas circunscripciones tienen una mayor probabilidad de votar al PP controlando por sus características individuales. El parámetro $\mu_{0 j}$ da cuenta de la variación entre circunscripciones del voto de los indecisos por el Partido Popular. Este parámetro es significativo al $95 \%$ por ciento de nivel de confianza en dos los modelos 1 y 2 de la tabla. En el resto, modelos 3 y 4 , es significativo tan solo a un $90 \%$ de nivel de confianza.

He introducido en los modelos una serie de variables agregadas de movilización para dar cuenta de esta variación entre circunscripciones. Ninguno de los coeficientes de las variables de movilización es significativo. Aun así es necesario resaltar que dos de los coeficientes de las variables contextuales -el indicador agregado de movilización y el de los mítines de Aznar- son positivos y bastante elevados. Los resultados de estas dos variables son coherentes con los del análisis bivariado. Los porcentajes de voto al PP aumentaban claramente en aquellas provincias visitadas por Aznar y donde el número de actividades movilizadoras realizadas había sido mayor. La coherencia con los resultados obtenidos en el análisis bivariado y el tamaño de los coeficientes nos hace pensar que estas dos 
Tabla 3.

El impacto de las estrategias de movilización del PP en el voto de todos los electores indecisos que decidieron votarles durante la campaña.

\begin{tabular}{|c|c|c|c|c|}
\hline $\begin{array}{l}\text { Variables } \\
\text { independientes }\end{array}$ & Modelo 1 & Modelo 2 & Modelo 3 & Modelo 4 \\
\hline \multicolumn{5}{|l|}{ Variables Actitudinales } \\
\hline Ideología & $\begin{array}{l}, 51^{*} \\
(, 22)\end{array}$ & $\begin{array}{l}, 52^{*} \\
(, 22)\end{array}$ & $\begin{array}{l}, 51^{*} \\
(, 22)\end{array}$ & $\begin{array}{l}, 54^{*} \\
(, 23)\end{array}$ \\
\hline Valoración de Aznar & $\begin{array}{l}31^{*} \\
(, 12)\end{array}$ & $\begin{array}{l}29 * \\
(, 11)\end{array}$ & $\begin{array}{l}33^{*} \\
(, 11)\end{array}$ & $\begin{array}{l}31^{*} \\
(, 11)\end{array}$ \\
\hline Representatividad & $\begin{array}{c}2,8^{* *} \\
(, 58)\end{array}$ & $\begin{array}{r}2,8 * * \\
(, 57)\end{array}$ & $\begin{array}{r}2,9 * * \\
(, 58)\end{array}$ & $\begin{array}{c}2,9 * * \\
(, 57)\end{array}$ \\
\hline \multicolumn{5}{|l|}{ Variables Sociodemográficas } \\
\hline Sin estudios & $\begin{array}{l}-1,1 \\
(, 69)\end{array}$ & $\begin{array}{l}-1,2 \\
(, 77)\end{array}$ & $\begin{array}{l}-1,2 \\
(, 77)\end{array}$ & $\begin{array}{l}-1,1 \\
(, 77)\end{array}$ \\
\hline Estudios primarios & $-1,7^{*}$ & $\begin{array}{l}-1,7^{*} \\
(, 76)\end{array}$ & $\begin{array}{l}-1,7^{*} \\
(, 62)\end{array}$ & $\begin{array}{l}-1,7^{*} \\
(, 75)\end{array}$ \\
\hline Bachillerato o FP & $\begin{array}{l}-1,1 \\
(, 72)\end{array}$ & $\begin{array}{l}-, 92 \\
(, 66)\end{array}$ & $\begin{array}{l}-92 \\
(, 69)\end{array}$ & $\begin{array}{l}-, 97 \\
(, 63)\end{array}$ \\
\hline$\mu_{0 \mathrm{j}}$ & $\begin{array}{l}1,4^{*} \\
(, 72)\end{array}$ & $\begin{array}{l}1,4^{*} \\
(, 73)\end{array}$ & $\begin{array}{l}1,7+ \\
(, 91)\end{array}$ & $\begin{array}{l}1,5+ \\
(, 81) \\
\end{array}$ \\
\hline \multicolumn{5}{|l|}{ Variables de Movilización } \\
\hline $\begin{array}{l}\text { Índice agregado de } \\
\text { movilización }\end{array}$ & $\begin{array}{c}, 64 \\
(, 41)\end{array}$ & & & \\
\hline Mítines del PP & & $\begin{array}{c}, 88 \\
(, 69)\end{array}$ & & \\
\hline $\begin{array}{l}\text { Inclusión de figuras relevantes las } \\
\text { candidaturas }\end{array}$ & & & $\begin{array}{c}, 59 \\
(, 71)\end{array}$ & \\
\hline Gasto provincial descentralizado & & & & $\begin{array}{l}-, 51 \\
(, 77)\end{array}$ \\
\hline Constante & & $\begin{array}{l}-5,8 \\
(, 22)\end{array}$ & $\begin{array}{c}-5,6 \\
(1,2)\end{array}$ & $\begin{array}{l}-5,3 \\
(1,2)\end{array}$ \\
\hline Número de casos & 652 & 652 & 652 & 652 \\
\hline
\end{tabular}

Fuente: CIS 2.210.

+ Significativo al $90 \%$. *Significativo al 95\%.**Significativos al $99 \%$.

Los coeficientes están calculados tomando como categoría de referencia a todos los que han cursado algún tipo de estudio universitario. 
RIS

estrategias de movilización tuvieron un cierto impacto, aunque no significativo, sobre el voto al PP en determinadas circunscripciones. El problema consiste en que aunque el tamaño de los coeficientes de estas dos variables sea elevado, el error estándar de ambos coeficientes también lo es. Esto indica una gran variación en el impacto de estas variables entre circunscripciones. Probablemente en algunas provincias el impacto de estas dos actividades es destacable mientras que en otras no. Para profundizar en el análisis del efecto de los mítines y el indicador agregado de movilización sobre el voto de los indecisos he realizado un análisis de los residuos por circunscripciones. De esta manera obtengo información adicional sobre el efecto de estas dos variables circunscripción por circunscripción. El gráfico de los residuos aparecen en las figuras 2 y 3 . Como puede observarse en la figura 2 hay varias circunscripciones cuya probabilidad de voto al PP es significativamente mayor que la media. Son las provincias de Sevilla, Valladolid, Murcia, Valencia, Palencia y Huesca. De estas provincias, el PP realizó mítines en las cuatro primeras. Podemos tener una mayor confianza en el coeficiente que mide la influencia de los mítines sobre el voto de los indecisos del PP en estas cuatro circunscripciones. Por el contrario, en otras como La Coruña, que corresponde a la observación situada más a la izquierda del gráfico de la figura 2, la probabilidad de votar al PP es significativamente menor que la media, a pesar de haber recibido la visita de Aznar. En esta provincia el impacto del mitin fue prácticamente nulo. En resumen, los resultados del análisis de residuos nos muestran que en algunas provincias como Sevilla, Valladolid o Valencia los mítines de Aznar sí influyeron en el voto de los indecisos que vivían en estas provincias. Por el contrario, en otras circunscripciones como La Coruna, no fue así. Esto explica que el coeficiente de la variable de mítines y de movilización sean elevados, pero a la vez su error estándar también lo sea.

El resto de las estrategias de movilización -inclusión de figuras populares del partido en las listas provinciales y gasto descentralizado - no tuvieron ninguna influencia en el voto de todos los indecisos, como puede verse en la tabla 3 en los modelos 3 y 4 . Los coeficientes son muy pequeños y claramente no significativos. Incluso el signo del coeficiente del gasto es negativo aunque claramente no significativo. Estos resultados son muy similares también a los obtenidos en el análisis bivariado. La inclusión de figuras relevantes en las listas tenía un efecto muy pequeño sobre el voto. Por su parte, el efecto del gasto descentralizado era el contrario al esperado.

En la tabla 4, se han estimado los mismos modelos de la tabla 3 pero para todos aquellos indecisos que decidieron votar durante la campaña y finalmente votaron al PP. Esto es, se ha filtrado el total de indecisos dejando sólo a aquellos que dudaban entre votar o abstenerse y finalmente votaron al PP Como era de esperar, y como sucedía con todos los indecisos, aquellos electores de derechas, que valoran positivamente Aznar y que se sienten representados por este partido tienen una mayor probabilidad de votarle. No obstante, y a diferencia de los resultados 
Figura 1.

Residuos por provincias del modelo 1

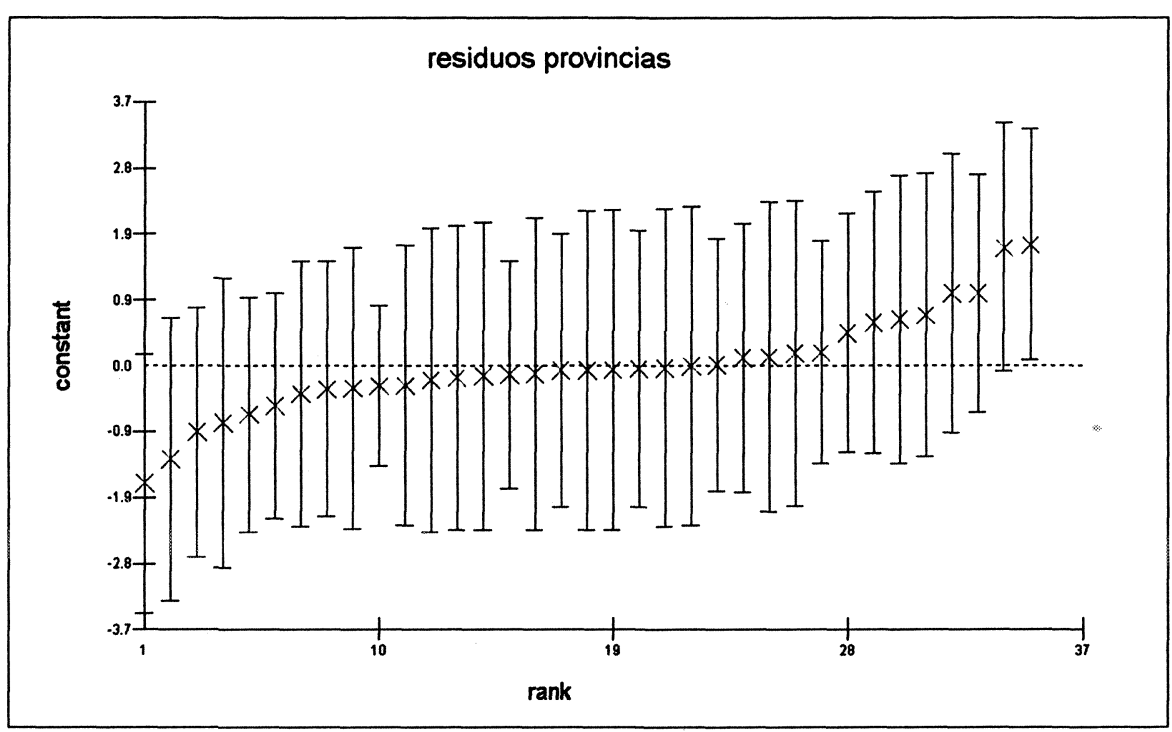

Figura 2.

Residuos por provincias del modelo 2

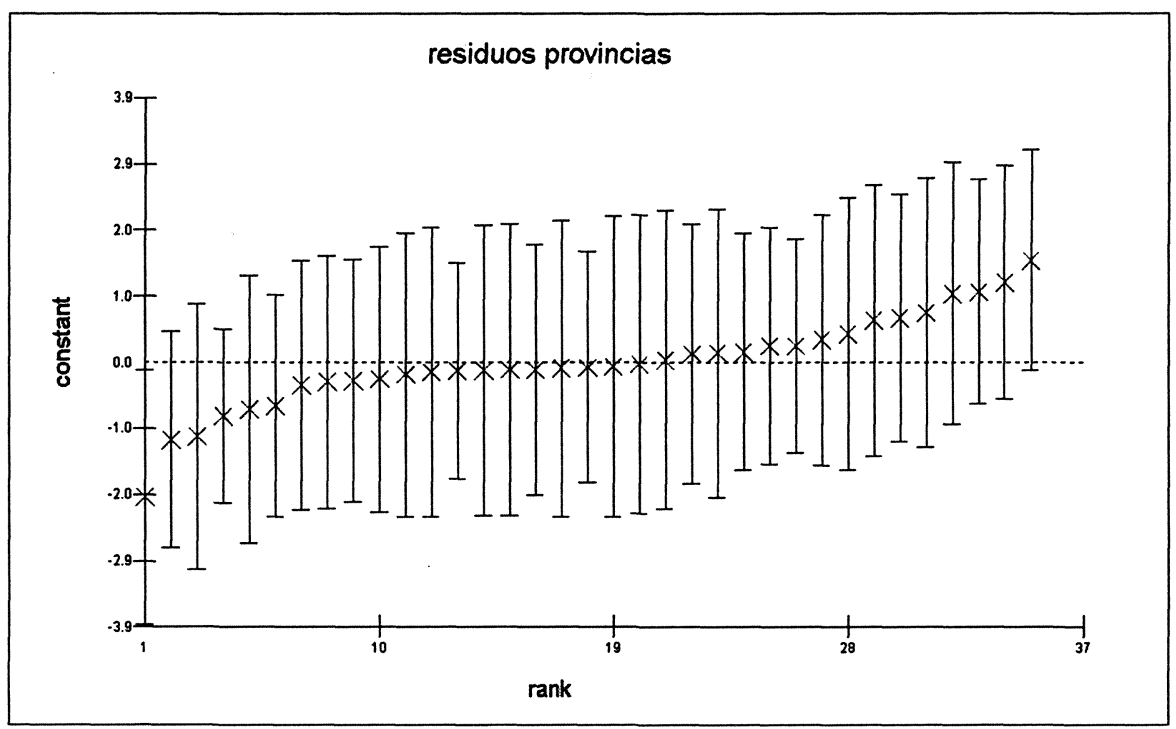


RIS

de la tabla 3, las categorías de educación no son significativas. La submuestra de indecisos que he utilizado en este segundo análisis tiene un tamaño menor que toda la muestra del total de indecisos. El número de electores en cada categoría de educación es bastante pequeño. Con pocos electores en cada categoría es más difícil que los coeficientes sean significativos. La micronumerosidad explica seguramente también la inestabilidad en los coeficientes de estas categorías en los distintos modelos.

Además de los efectos de las variables individuales, lo más interesante de estos resultados son los resultados de las variables de movilización. Como puede observarse, todos los coeficientes de las variables de movilización son positivos, aunque sólo el indicador agregado de movilización es significativo. Los indecisos que residían en circunscripciones donde el PP había realizado varias actividades movilizadoras tienen una probabilidad mayor de votarle manteniendo cónstantes sus características individuales. Como se trata de modelos logísticos, los coeficientes no son directamente interpretables. Por ello, he calculado las probabilidades predichas del modelo dejando variar la ideología y manteniendo constante en sus medias el resto de las variables del modelo (Long, 1997: 64-70). He generado dos diferentes escenarios. En el primero de ellos he calculado cómo aumenta la probabilidad de votar al PP a medida que varía su ideología de un elector identificado con el PP, que valora Aznar con un 5 y que vive en una circunscripción movilizada. En el segundo escenario he calculado cómo cambia esta probabilidad para el mismo elector que vive en una circunscripción no movilizada.

En la figura 3 se muestran las probabilidades predichas de ambos escenarios. Como puede observarse, la probabilidad de un elector indeciso de centro derecha (autoubica en el 6) de votar por el PP en una circunscripción movilizada es de 0,56 . La probabilidad de que este mismo elector votase por el PP si vive en una circunscripción no movilizada desciende hasta .34. La movilización aumenta en un $22 \%$ la probabilidad de votar por el PP. Este porcentaje puede resultar crucial en aquellas circunscripciones donde un escaño es competido. Puede significar la diferencia entre ganarlo o perderlo.

Al comparar los resultados obtenidos en las tablas 3 y 4 se observa, en primer lugar, que las variables de movilización territorial más eficaces en la obtención de votos fueron los mítines realizados por Aznar y el indicador agregado de movilización, que es una suma de todas ellas. En segundo lugar, es destacable también que las variables de movilización territorial tienen un mayor impacto en los indecisos que dudaban entre votar y abstenerse que en los indecisos en su totalidad. De hecho, el impacto de la movilización, en concreto el indicador agregado, sólo es significativo para este perfil de electores. Esto significa que la movilización tiene fundamentalmente un efecto activador del voto. La movilización territorial aumenta significativamente la probabilidad de votar de aquellos electores que dudaban entre votar $o$ abstenerse. No tiene un efecto significativo, sin embargo, entre todos los indecisos. Esto se debe a que este grupo lo forman tanto aquellos 


\section{Tabla 4.}

El impacto de las estrategias de movilización del PP en los votantes indecisos que decidieron a qué partido votar durante la campaña.

\begin{tabular}{|c|c|c|c|c|}
\hline Variables independientes & Modelo 1 & Modelo 2 & Modelo 3 & Modelo 4 \\
\hline \multicolumn{5}{|l|}{ Variables Actitudinales } \\
\hline Ideología & $\begin{array}{l}, 89^{*} \\
(, 28)\end{array}$ & $\begin{array}{l}91^{*} \\
(, 22)\end{array}$ & $\begin{array}{l}, 86^{*} \\
(, 22)\end{array}$ & $\begin{array}{l}, 82^{*} \\
(, 28)\end{array}$ \\
\hline Valoración de Aznar & $\begin{array}{l}, 32 * \\
(, 16)\end{array}$ & $\begin{array}{l}31^{*} \\
(, 11)\end{array}$ & $\begin{array}{l}33 * \\
(, 11)\end{array}$ & $\begin{array}{l}, 33^{*} \\
(, 16)\end{array}$ \\
\hline Representatividad & $\begin{array}{c}2,8^{* *} \\
(, 68)\end{array}$ & $\begin{array}{c}2,8^{* *} \\
(, 57)\end{array}$ & $\begin{array}{c}2,5^{* *} \\
(, 61)\end{array}$ & $\begin{array}{c}2,5 * * \\
(, 64)_{*}\end{array}$ \\
\hline \multicolumn{5}{|l|}{ Variables Sociodemográficas } \\
\hline Sin estudios & $\begin{array}{l}-, 27 \\
(, 79)\end{array}$ & $\begin{array}{l}-, 12 \\
(, 77)\end{array}$ & $\begin{array}{c}-, 01 \\
(, 787)\end{array}$ & $\begin{array}{l}-, 217 \\
(, 95)\end{array}$ \\
\hline Estudios primarios & $\begin{array}{l}-, 38 \\
(, 89)\end{array}$ & $\begin{array}{l}, 11 \\
(, 76)\end{array}$ & $\begin{array}{l}, 10 \\
(, 62)\end{array}$ & $\begin{array}{l}0,09 \\
(, 75)\end{array}$ \\
\hline Bachillerato o FP & $\begin{array}{l}-, 34 \\
(, 57)\end{array}$ & $\begin{array}{l}, 10 \\
(, 66)\end{array}$ & $\begin{array}{c}, 04 \\
(, 69)\end{array}$ & $\begin{array}{l}-, 05 \\
(, 63)\end{array}$ \\
\hline \multicolumn{5}{|l|}{ Variables de Movilización } \\
\hline Índice agregado de movilización & $\begin{array}{l}93 * \\
(, 45)\end{array}$ & & & \\
\hline Mítines del PP & & $\begin{array}{l}, 79 \\
(, 69)\end{array}$ & & \\
\hline $\begin{array}{l}\text { Inclusión de figuras relevantes las } \\
\text { candidaturas }\end{array}$ & & & $\begin{array}{c}, 69 \\
(, 71)\end{array}$ & \\
\hline Gasto provincial descentralizado & & & & $\begin{array}{c}, 04 \\
(, 06)\end{array}$ \\
\hline Constante & $\begin{array}{c}-10,0^{* *} \\
(, 01)\end{array}$ & $\begin{array}{c}-8,9 * * \\
(2,3)\end{array}$ & $\begin{array}{c}-5,6^{* *} \\
(1,2)\end{array}$ & $\begin{array}{l}-8,3^{* *} \\
(1,9)\end{array}$ \\
\hline Número de casos & 523 & 523 & 523 & 523 \\
\hline
\end{tabular}

Fuente: CIS 2210.

+ Significativo al $90 \%$. *Significativo al $95 \%$. **Significativos al $99 \%$.

Los coeficientes están calculados tomando como categoría de referencia a todos los que han cursado algún tipo de estudios universitarios.

que dudaban entre votar o abstenerse como los electores que dudaban por qué partido votar. A la luz de los resultados, las estrategias de movilización de los partidos no tienen un efecto conversor de las preferencias. Simplemente, activan las preferencias existentes. 
RIS

REVISTA INTERNACIONAL DE SOCIOLOGIA

NN 32, Mayo-Agosto, 2002

Figura 4.

Probabilidad de votar al PP de los indecisos (aquéllos que decidieron votar durante la campaña y votaron finalmente al PP).

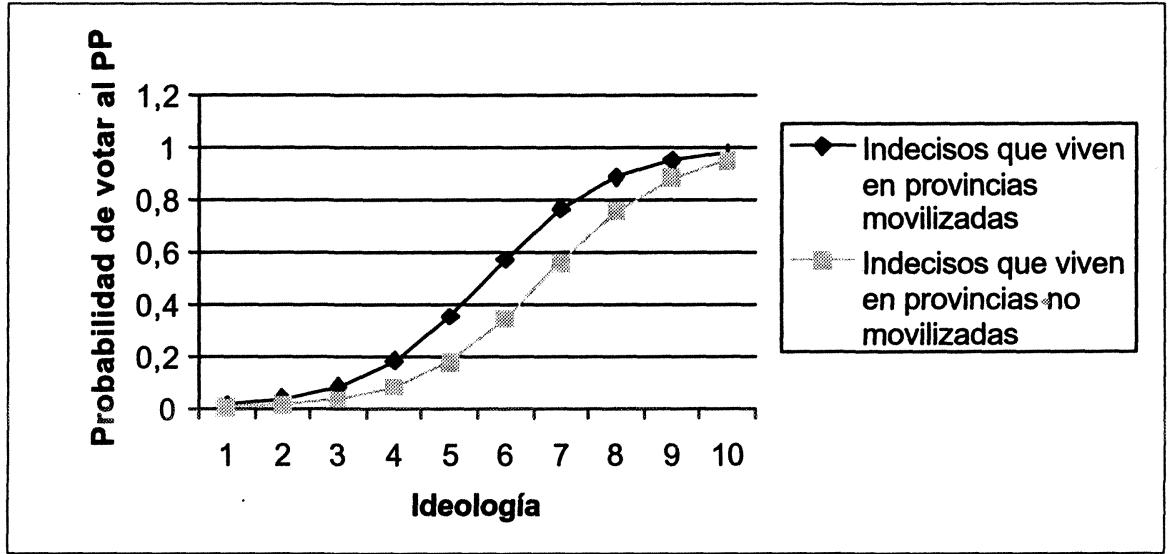

\section{CONCLUSIONES}

En este artículo he intentado responder a la pregunta planteada en la introducción: ¿tienen las estrategias de movilización territorial de los partidos (visitas del candidato, gasto por circunscripción) efectos sobre el voto de los electores? A la luz de los resultados obtenidos podemos responder que las visitas del candidato, en este caso Aznar, y el indicador agregado de movilización sí incrementaron la probabilidad de votar por el PP, aunque este incremento sólo fue significativo para los votantes indecisos que dudaban entre el voto y la abstención. De esto se deduce que la movilización territorial tiene, fundamentalmente, un efecto de activación del voto. A estas conclusiones se ha llegado estimando modelos logit multinivel. Estos modelos permiten estimar los efectos de las variables contextuales - en este caso las de movilización - controlando el efecto de las características individuales de los electores. Los resultados obtenidos son, por ello, bastante robustos.

\section{REFERENCIAS}

ANDUIZA PEREA, E. (1999), ¿Individuos o Sistemas?: la abstención en Europa Occidental, Centro de Investigaciones Sociológicas, Madrid.

CAMPBELL, A., Ph.E. CONVERSE, W.E. MILLER y D.E. STOKES (1960), The American Voter, Nueva York, John Wiley and sons. 
CAÍNZOS, M. A. (2001), "La evolución del voto clasista en España", Zona Abierta, n 96/97, pp. 91-171.

COX, G.W. y C. M. MUNGER (1989), "Closeness, Expenditures, and Turnout in the 1982 U.S. House Elections", American Political Science Review, n 83 (1), pp. 217-230.

COX, G.W., F. M. ROSENBLUTH, y M. F. THIES (1998), "Mobilization, Social Networks, and Turnout. Evidence from Japan", World Politics, n 50, pp. 447-474.

CRIADO, H. (2002), "Strategic Mobilization under Uncertainty. The Logic of PSOE Mobilization Strategies in the 1996 Spanish Electoral Campaign", Estudio/Working Paper 2002/181, Madrid, Instituto Juan March de Estudios e Investigaciones.

GOLDSTEIN, H. (1995), Multilevel Statiscal Models, Londres, Edward Arnold.

GOLDSTEIN, K. (1999), Watering the Grassroots. Interest Groups, Lobbiying and Participation, Ann Arbor, Michigan University Press.

GONZÁLEZ, J. J. (1996), “Clases, ciudadanos y clases de ciudadanos. El ciclo electoral del postsocialismo (España, 1986-1994)", Revista Española de Investigaciones Sociológicas, $\mathrm{n}^{\circ}$ 97, pp. 125-155.

GUJARATI, D. (1997), Econometria, Colombia, McGraw-Hill.

GUNTHER, R. y J.R. MONTERO (1994), "Los anclajes del partidismo: un análisis comparado del comportamiento electoral en cuatro democracias del sur de Europa”, en Pilar del Castillo (coord.), Comportamiento electoral y politico, Madrid, Centro de Investigaciones Sociológicas.

HOLBROOK, T.M. (1996), Do Campaigns Matter?, Londres, Sage Publications.

HUCKFELDT, R. y J. SPRAGUE (1992), "Political Parties and Electoral Mobilization: Political Structure, Social Structure, and the Party Canvass", American Political Science Review, $\mathrm{n}^{\circ} 86$, pp. 70-86.

HUCKFELDT, R., P. ALLEN BECK y R. DALTON (1994), Partisan Comunication and Mobilization in the 1992 Presidential election, Manuscrito no publicado.

JACKMAN, R. W. (1987), "Political Institutions and Voter Tournout in the Industrial Democracies", American Political Science Review, $\mathrm{n}^{\circ}$ 81, pp. 405-23.

JONES, K. y N. BULLEN (1994), "Contextual Models of Urban Houses Prices: a Comparison of Fixed and Random Coefficients Models Developed by Expansions", Economic Geography, n ${ }^{\circ}$ 70, pp. 252-270.

JOHNSON, M., P. SHIVELY y R.M. STEIN (2002), "Contextual Data and the study of elections and voting behavior", Electoral Studies, ${ }^{\circ} 21$, pp. 219-233.

LAZARSFELD, P.F., B. BRELSON y H. GAUDET (1948), The People's Choice, Nueva York, Columbia University Press. 
RIS

REVISTA INTERNACIONAL DE SOCIOLOCII

No 32, Mayo-Agosto, 2002

HENAR CRIADO OLMOS

LIPSET, S. M. y S. ROKKAN (1967), "Cleavages structures, Party Systems, and Voter alignments. An Introduction", en S.M. Lipset y S. Rokkan (eds.), Party Systems and Voter Alignments: crossnational perspectives, Nueva York, Free Press.

LONG, J. S. (1997), Regression Models for Categorical and Limited Dependent Variables, Londres, Sage.

MARSH, M. (2002), “Electoral Context”, Electoral Studies, n² 21, pp. 207-217.

PEÑA, D. (2001), Fundamentos de Estadística, Madrid, Alianza Editorial.

POPKIN, S. (1991), The Reasoning Voter, Chicago, The University Of Chicago Press.

ROSENSTONE, S.J. y M.J. HANSEN (1993), Mobilization, Participation and Democracy in America, Nueva York, Macmillan.

STOKER, L.y J. BOWERS, (2002), "Designing Multilevel Studies: Sampling Voters and Electoral Contexts", Electoral Studies, ${ }^{\circ}$ 21, pp. 235-267.

TORCAL, M. y P. CHHIBBER (1997), "Elite Strategy, Social Cleavage, and Party Systems in a New Democracy. Spain", Comparative Political Studies, n 30, pp. $27-54$.

VERBA, S., N. NORMAN y J. KIM, (1978), Participation and Political Equality, Nueva York, Cambridge University Press.

WIELHOUWER, P. y B. LOCKERBIE (1994), "Party Contacting and Political Participation 195290", American Journal of Political Participation, vol. 38, pp. 211-229.

WOLFINGER, R. y S.J. ROSENSTONE, (1980), Who Votes?, New Haven, Yale University Press. 